II Congresso Brasileiro de

Fluidodinâmica Computacional

27 a 29 de Junho de 2018

Rio de Janeiro, RJ

\title{
MODELAGEM NÚMERICA DO CAMPO DE TENSÃO DE CISALHAMENTO DO SOLO NO ENSAIO DE PENETRÔMETRO DE PIEZOCONE ATRAVÉS DO MODELO HERSCHEL-BULKLEY
}

\author{
G. M. S. AYRES ${ }^{1}$ e R. J. LOBOSCO ${ }^{1}$ \\ ${ }^{1}$ Universidade Federal do Rio de Janeiro \\ E-mail para contato: gabrielmsa@uol.com.br; rlobosco@macae.ufrj.br
}

\begin{abstract}
RESUMO - Apesar dos avanços recentes nos métodos numéricos, a modelagem de deformação do solo ainda é um problema de grande complexidade. O presente artigo avalia as tensões de penetração no solo em um ensaio de um penetrômetro de piezocone. O campo de tensões de cisalhamento de um fluido não-newtoniano do tipo Herschel-Bulkley, com reologia semelhantes a da lama, é avaliado e os efeitos viscosos são comparados com o limite de liquidez do solo.
\end{abstract}

\section{INTRODUÇÃO}

As propriedades do solo, necessárias para caracterização geológica de fundações profundas, são estimadas a partir de dados experimentais e ensaios de campo. O teste de penetrômetro de piezocone é uma importante ferramenta que permite a coleta de dados de um solo in situ.

Estudos desse ensaio foram realizadas anteriormente por Markauskas et al. (2005). A análise numérica foi baseada no método de elementos finitos considerando o solo como um meio poroso. Métodos alternativos para a geração da malha em simulações desse tipo foram propostas por Moavenian et al. (2016). Na investigação de um fluido composto de uma mistura de água e caulinita em diferentes concentrações, Maciel et al. (2009) mostraram uma boa aproximação entre o modelo Hershel-Bulkley e os dados experimentais.

\section{MÉTODOS NUMÉRICOS}

O estudo experimental das tensões no solo é feito por meio do uso de um penetrador in situ que perfura o solo à uma taxa de $1 \mathrm{a} 2 \mathrm{~cm} / \mathrm{s}$ e coleta dados. Em uma análise de campo Januzzi et al. (2015) efetuou a caracterização do solo da área de Sapucaí II. Esse procedimento foi reproduzido computacionalmente de forma que os dados experimentais pudessem ser comparados com os obtidos experimentalmente. As análises do modelo reológico são comparados com os dados de Maciel et al. (2009).

Um dos problemas atrelados a simulação de testes de penetração é a remalhagem devido a deformação do solo (Markauskas et al., 2005). A malha do domínio computacional do fluido foi feita de tal forma que ele ficasse compreendido entre um cilindro com 75 milímetros de diâmetro e 481,2 milímetros de altura, e o penetrador de piezocone, com 35,7 


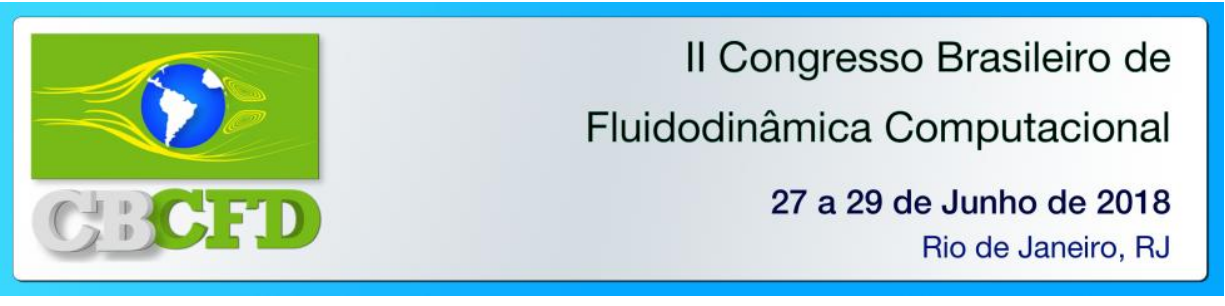

milímetros de diâmetro, 100 milímetros de altura e com uma angulação de $60^{\circ}$ na ponta. As paredes do penetrador foram definidas como sólido rígido enquanto as laterais, partes superior e inferior do cilindro foram definidas como condição de entrada ou saída generalizadas. A Figura 1 mostra um corte transversal feito na geometria utilizada e as Figura 2, 3 e 4 mostram a malha em diferentes vistas.

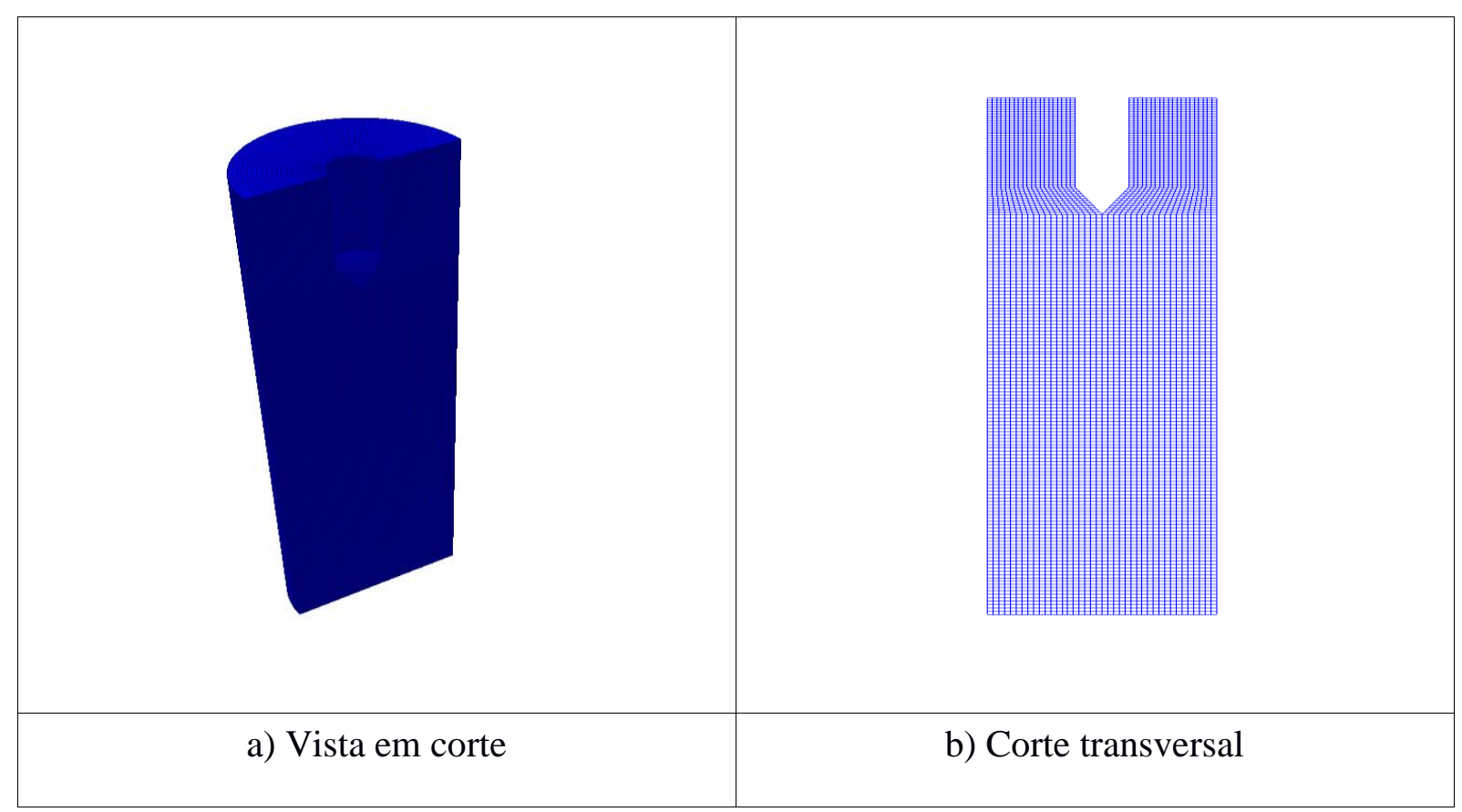

Figura 1 - O domínio computacional

Duas simulações com diferentes condições iniciais foram realizadas. Na simulação A foi definida uma velocidade de fluido de $2 \mathrm{~cm} / \mathrm{s}$ no penetrador enquanto na simulação $\mathbf{B}$ a velocidade de $2 \mathrm{~cm} / \mathrm{s}$ foi imposta no fluido, sendo definida uma velocidade nula no penetrador. As outras fronteiras foram definidas com condições de contorno de Neumann.

O modelo reológico de Herschel-Bulkley pode ser definido conforme a Equação 1.

$$
\tau=\tau_{0}+k(\gamma)^{n}
$$

Em que: $\tau=$ tensão cisalhante, $\tau_{0}=$ tensão mínima necessária para início da deformação, $\mathrm{k}=$ fator de consistência, $\gamma=$ deformação, $\mathrm{n}=$ índice de escoamento (responsável por representar uma deformação não linear com a tensão quando diferente de 1).

Os parâmetros reológicos do fluido foram definidos conforme Maciel et al. (2009) e encontram-se descritos na Tabela 1.

Tabela 1 - Parâmetros utilizados no modelo reológico, conforme descritos em Maciel et al. (2009) 


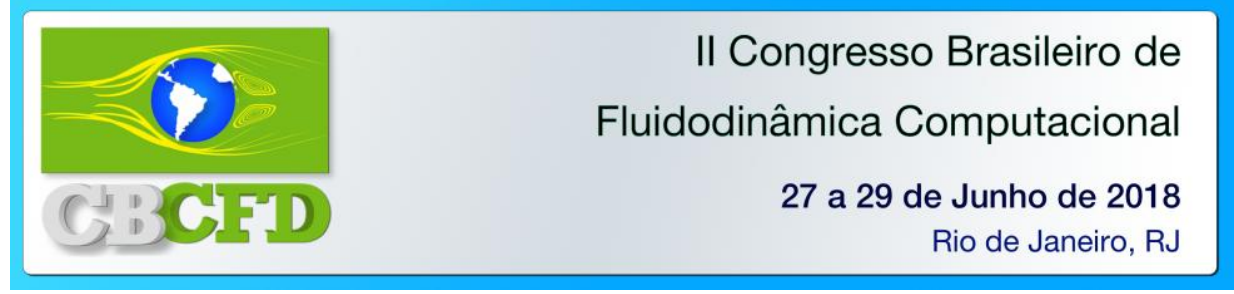

\begin{tabular}{lcccc}
\hline $\mathrm{C}_{\mathrm{v}}(\%)$ & $\tau_{\mathrm{c}}(\mathrm{Pa})$ & $\mathrm{k}\left(\mathrm{Pas}^{\mathrm{n}}\right)$ & $\mathrm{n}$ & $\rho\left(\mathrm{kg} / \mathrm{m}^{3}\right)$ \\
\hline 10 & 1,26 & 0,22 & 0,47 & 1165 \\
\hline
\end{tabular}

Os parâmetros utilizados foram os correspondentes a mistura com $10 \%$ de concentração de caulinita, cuja massa específica é $1165 \mathrm{~kg} / \mathrm{m}^{3}$.

\section{RESULTADOS E DISCUSSÕES}

A coleta de dados da tensão foi feita na ponta do piezocone. As Figuras $2 \mathrm{a}$ e $2 \mathrm{~b}$ mostram a evolução da tensão $\left(\mathrm{em} \mathrm{m}^{2} / \mathrm{s}^{2}\right)$, neste ponto, ao longo do tempo, para a simulação A e B, respectivamente. É possível notar que a tensão sai de um ponto muito alto e decai rapidamente, sofrendo um lento incremento com o tempo a seguir. Esse rápido decaimento se deve ao cálculo da viscosidade do fluido, conforme demonstra a Equação 2.

$$
v=\min \left(v_{0}, \frac{\tau_{0}}{\dot{\gamma}}+k \dot{\gamma}^{\mathrm{n}-1}\right)
$$

Em que: $v=$ viscosidade cinemática, $\min ()=$ é a função mínimo, $v_{0}=$ viscosidade cinemática para taxas de deformação muito baixas e $\dot{\gamma}=$ taxa de deformação.

É necessário fornecer um valor para a viscosidade inicial, $v_{0}$, que é utilizado para o cálculo das tensões quando a taxa de deformação $\dot{\gamma}$ é pequena demais para ultrapassar a tensão de cisalhamento $\tau_{0}$. Assim, foi definido um valor alto inicial para que, com o incremento da taxa de deformação, fosse calculada a viscosidade com base nos parâmetros de Herschel-Bulkley definidos na Tabela 1.

Devido a alta variabilidade dos valores da tensão, mas levando-se em conta a tendência das curvas, foi calculado o valor médio da magnitude da tensão $\left(\mathrm{m}^{2} / \mathrm{s}^{2}\right)$ multiplicado pelo valor da massa específica, resultando em valores de tensão em Pa.Para a simulação A o valor encontrado foi de 1,31 Pa e na simulação $\mathbf{B}$ foi de 1,97 Pa, valores condizentes com os encontrados por Maciel et al. (2009).

\section{CONCLUSÕES}

Foram realizados testes com o modelo de fluido Hershel-Bulkley sob diferentes condições de contorno para a representação de um ensaio de penetração no solo do tipo piezocone. Este trabalho busca progredir com o entendimento da influência da forças viscosas no limite de escoamento do solo. A partir de uma análise de tensões é possível definir o campo de tensões cisalhantes em todo o domínio do escoamento. 

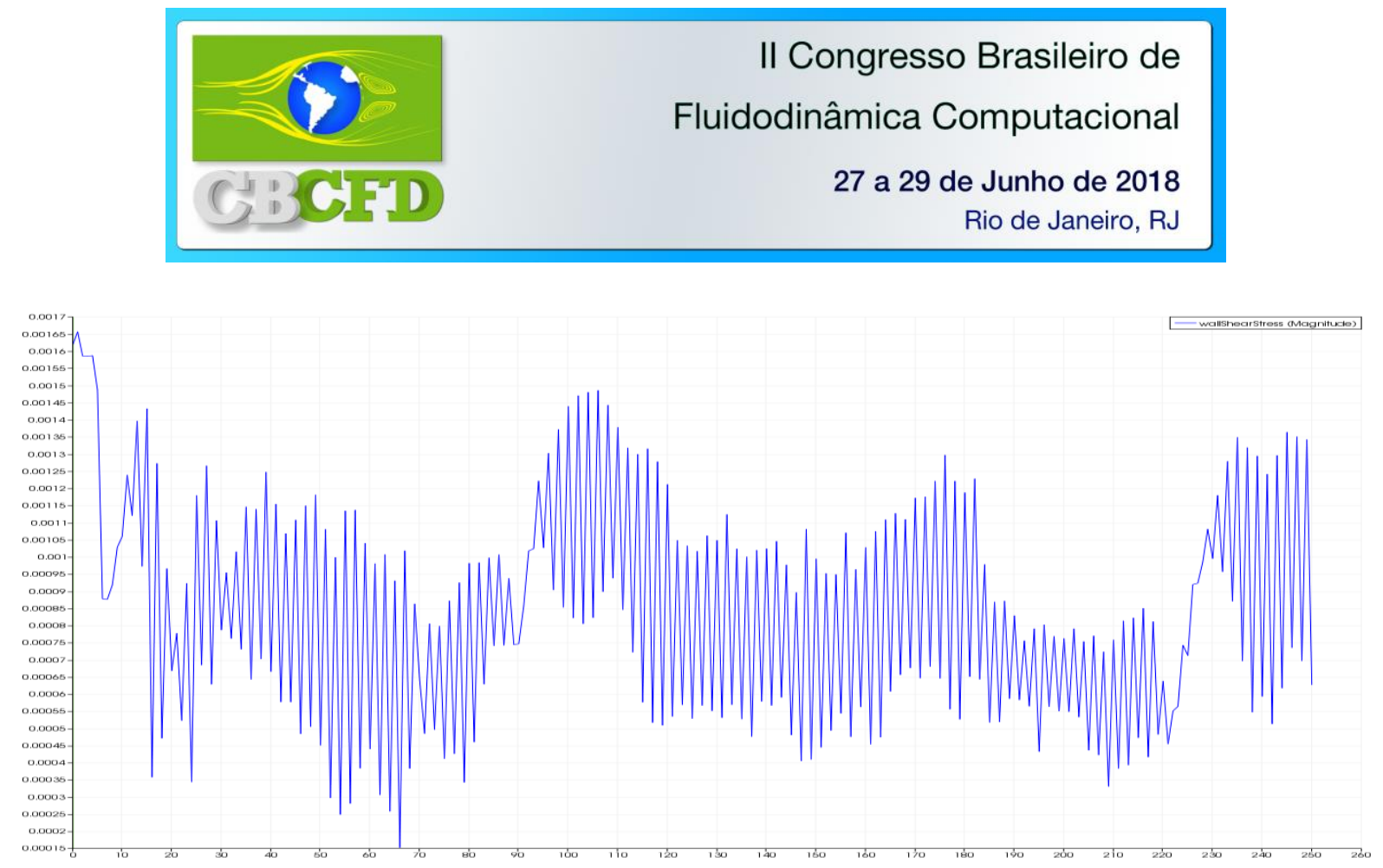

Figura $\underline{2 \mathrm{a}}$ - Evolução da tensão com o tempo na simulação A

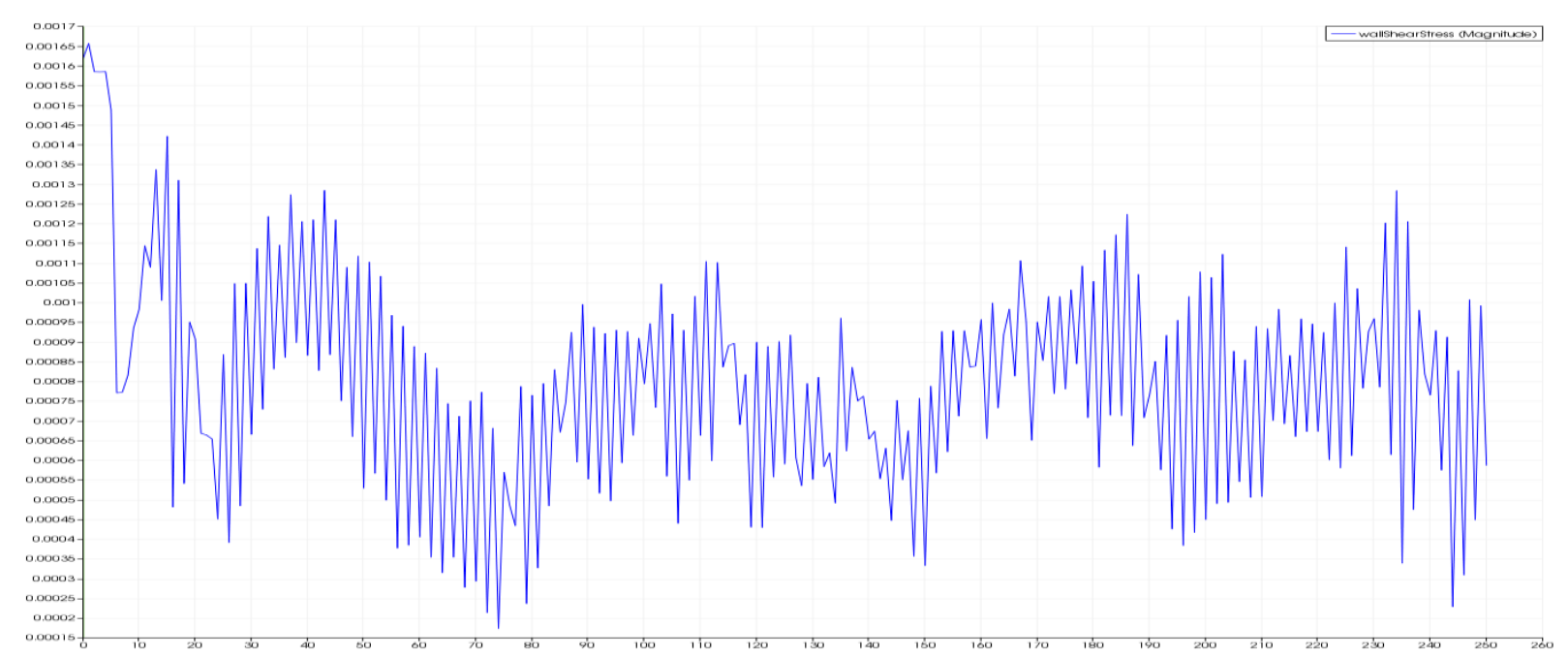

Figura 2b - Evolução da tensão com o tempo na simulação B

\section{REFERÊNCIAS BIBLIOGRÁFICAS}

JANNUZZI, G.; DANZIGER, F.; MARTINS, I. Geological-geotechnical characterisation of Sarapuí II clay, 2015.

MACIEL, G.; SANTOS, H.; FERREIRA, F. Rheological analysis of water clay compositions in order to investigate mudflows developing in canals, 2009.

MARKAUSKAS, D.; KACIANAUSKAS, R.; KATZENBACH, R. Simulation of piezocone penetration in saturated porous medium using the FE remeshing technique, 2005.

MOAVENIAN, M.H.; NAZEM, M.; CARTER, J.P. Alternative mesh refinement methods for analysing soil penetration problems, 2016. 